\title{
Usefulness of Elastography for the Evaluation of Subcentimeter Solid Breast Nodules
}

\author{
Florentina Guzmán-Aroca ${ }^{1,2, *}$, Yésica Martínez-Paredes ${ }^{3}$, Juan de Dios Berná-Serna ${ }^{1,2}$, \\ Ana Azahara García-Ortega 1,2, Juan de Dios Berná-Mestre ${ }^{1,2}$ and Miguel Alcaraz ${ }^{2,4, *}$
}

1 Department of Radiology, Hospital Clínico Universitario "Virgen de la Arrixaca”, Ctra. Madrid-Cartagena, 30120 Murcia, Spain; jdberna@um.es (J.d.D.B.-S.); anaazahara.garcia@um.es (A.A.G.-O.); juandeberna@um.es (J.d.D.B.-M.)

2 Instituto Murciano de Investigación Biosanitaria "Virgen de la Arrixaca" (IMIB-Arrixaca), 30120 Murcia, Spain

3 Department of Radiology, Hospital Universitario "Santa Lucía", C/Mezquita, s/n, Paraje Los Arcos, 30202 Murcia, Spain; yesica.martinez@um.es

4 Radiology and Physical Medicine Department, School of Medicine, University of Murcia, 30100 Murcia, Spain

* Correspondence: florentina.guzman@um.es (F.G.-A.); mab@um.es (M.A.); Tel.: +34-968-381-188 (F.G.-A.); +34-868-883-601 (M.A.)

Citation: Guzmán-Aroca, F.

Martínez-Paredes, Y.; de Dios

Berná-Serna, J.; García-Ortega, A.A.; de Dios Berná-Mestre, J.; Alcaraz, M. Usefulness of Elastography for the Evaluation of Subcentimeter Solid Breast Nodules. Appl. Sci. 2021, 11, 1409. https://doi.org/10.3390/app 11041409

Academic Editor: Caterina Ledda

Received: 29 December 2020

Accepted: 1 February 2021

Published: 4 February 2021

Publisher's Note: MDPI stays neutral with regard to jurisdictional claims in published maps and institutional affiliations.

Copyright: (c) 2021 by the authors. Licensee MDPI, Basel, Switzerland. This article is an open access article distributed under the terms and conditions of the Creative Commons Attribution (CC BY) license (https:// creativecommons.org/licenses/by/ $4.0 /)$.
Abstract: The accurate diagnosis of subcentimeter lesions is controversial, and therefore a standardized diagnosis algorithm is needed. The objective of the present work was to study the value of the elastography patterns obtained through the use of the shear wave elastography (SWE) technique with respect to histopathology for the evaluation of nodular breast lesions $\leq 1 \mathrm{~cm}$. A retrospective study was conducted which included 65 sub-centimeter lesions from 57 patients with an average age of $45.6 \pm 11.9$. For all the cases, a B-mode ultrasound study, shear wave elastography, and a posterior anatomopathological study were conducted. The lesions had a diameter greater than $7.5 \pm 1.7 \mathrm{~mm}$ (range: 4-9 mm). Through elastography, the distribution of the patterns was: cyst artifact $(\mathrm{n}=13)$, pattern $1(n=4)$, pattern $2(n=31)$, pattern $3(n=13)$, and pattern $4(n=4)$. Of the 65 lesions, 15 were cysts, 46 were solid benign lesions, and 3 were malignant lesions. The sensitivity of the elastography was $75 \%$, with a specificity of $98.46 \%$ and a correct diagnosis in $96.92 \%$ of the cases $(n=63)$. The results from this study show the usefulness of SWE for the evaluation of sub-centimeter breast lesions. In addition, this diagnostic strategy helps with the differential diagnosis between benign and malignant lesions and contributes to the early detection of malignant breast lesions.

Keywords: breast; elastography; ultrasound; shear wave imaging

\section{Introduction}

Elastography is a type of imaging method based on the stiffness of a tissue, which is analogous to a clinical palpation for a malignant neoplasm, as it provides a quantification of stiffness [1], with ultrasound elastography (USE) first being described in the 1990s [2]. Elastography maps show additional information in B-mode, as with characterization via Doppler, thus increasing the diagnostic precision [3-6].

The two types of elastography utilized with breasts are compression elastography (strain imaging) and shear wave elastography (SWE). These techniques are included in the 5th edition of the Breast Imaging Reporting and Data System (BI-RADS ${ }^{\circledR}$ ) [7] without specifying the management algorithm ("If a lesion was evaluated with color or power Doppler or with strain or shear-wave elastography, observations relevant to the interpretation should be reported"), and both of them are approved by the Food and Drug Administration (FDA). The greater advantage of elastography is the improvement in the characterization of BI-RADS 3 and 4 a lesions, so that we are able to "upgrade" or "downgrade" the lesions as a function of the elastography maps provided by the image [8], in a similar manner as that provided by diffusion in magnetic resonance in the study of the prostate. It could be considered an image biomarker. 
At present, there are very few studies that deal with the contribution of elastography to the management of small-sized nodules [9-11], and we found no studies which dealt with nodules smaller than $1 \mathrm{~cm}$. These lesions, due to their small size, are difficult to characterize and tend to force the radiologist to perform unnecessary controls or biopsies, and can even induce errors in their classification. In the management of these lesions, elastography could be helpful for establishing a differential diagnosis between benign and malignant breast lesions. The objective of this study was to investigate the value of grayscale elastography patterns generated through the semiquantitative elastography (SW) technique, with respect to results obtained from the anatomopathological study of nodular lesions measuring $\leq 1 \mathrm{~cm}$.

\section{Patients and Methods}

\subsection{Patients}

A retrospective study was conducted from August 2018 to March 2019, with 60 patients who had lesions $\leq 1 \mathrm{~cm}$, which were detected with an ultrasound (US) in women who were referred from the Breast Care Unit at our hospital. A conventional US was performed in all the patients to evaluate the possible lesions. The US findings were categorized according to the American College of Radiology Breast Imaging Reporting and Data System $\left(\right.$ BI-RADS $^{\circledR}$ ) [7]. When nodules smaller than one centimeter were detected during the examination, which showed characteristics of solid lesions, the study was complemented with elastography, obtaining an grayscale elastogram as well as a cytological or histological analysis in BI-RADS 3 lesions as an alternative to short-term follow-up and the same for BI-RADS 4, or through a fine needle aspiration (FNA) if the finding was defined as a BI-RADS 3, or with a core needle biopsy (CNB) or vacuum-assisted biopsy (VAB) if it was defined as a BI-RADS 4 lesion (as a function of the elastography results). The study was approved by the Institutional Review Board. In all cases, the procedure was explained in detail to the patient and informed consent was obtained.

The inclusion criteria of the study were the US detection of solid nodular lesions $\leq 1 \mathrm{~cm}$ analyzed through an ultrasonography and an elastography, and those who were subjected to FNA or CNB/VAB. The patients were excluded if they had lesions measuring $\geq 1 \mathrm{~cm}$, and those who had a $\leq 1 \mathrm{~cm}$ lesion that was benign according to the US (BI-RADS 2), and lesions that were not studied through histopathological studies (BI-RADS 3 lesions with a recommendation of short-term/6-month follow-up).

\subsection{Evaluation with Elastography}

For the ultrasonography evaluation, an Acuson S2000 (Siemens AG, Erlangen, Germany) was utilized, with a linear probe of 18L6 Mhz for the acquisition of the B-mode image (conventional), and a linear probe of 9L4 MHz for the elastography study. The elastography study was conducted with the Virtual Touch, which provided elastograms through a grayscale map with the SWE technique. Elastograms were obtained from all the nodules, and the results were classified according to the model by Tsokuba adapted to the grayscale map [12], as shown in Figure 1.

- Pattern 1: Elastogram with a deformable homogenous pattern (not stiff).

- Pattern 2: Elastogram with a mosaic pattern (with deformable and non-deformable areas).

- Pattern 3: Elastogram with a pattern in which a deformable peripheral area (not stiff) with a less deformable central area (more stiff) is observed.

- Pattern 4: Elastogram with a non-deformable (stiff) homogeneous pattern with a lesion periphery with normal characteristics.

- Pattern 5: Elastogram with non-deformable homogeneous pattern (stiff) with a nondeformable lesion periphery.

On the other hand, the cyst artifact result was considered on its own. When this result was found, the Tsokuba model was not utilized. 


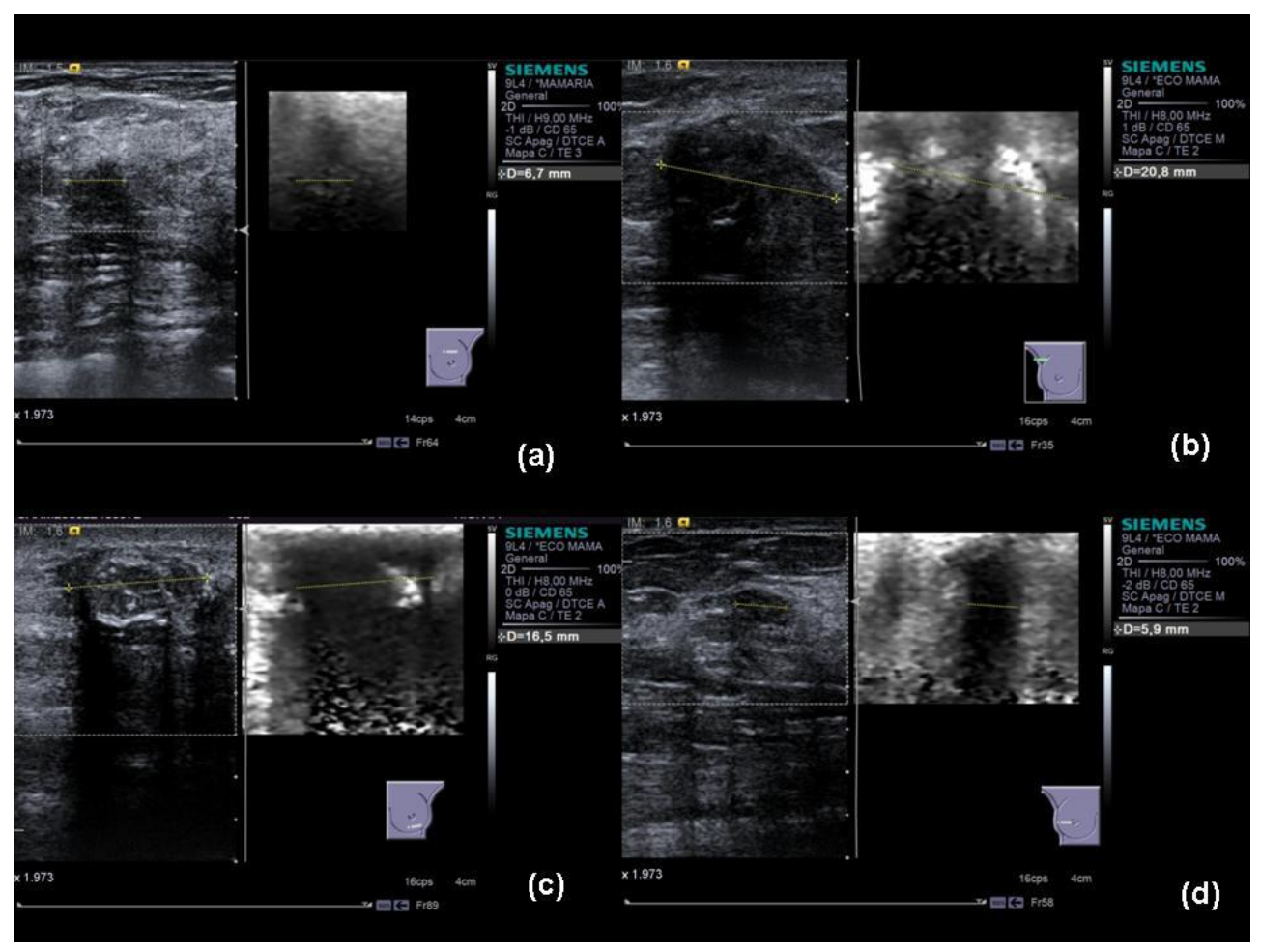

Figure 1. Shear wave elastography patterns. Pattern 1 (a) - a pattern of nodular elasticity similar to adjacent tissue. Pattern 2 (b) - a pattern of elasticity that is soft in most of its area. Pattern 3 (c) - a pattern of elasticity that is stiff in most of its area. Pattern 4 (d) - a pattern of elasticity that is stiff over the entire nodule without exceeding the limits of the lesion.

\subsection{Histopathological Evaluation}

The sample was obtained through echo-guided punctures (US-guided) performed by a radiologist who was an expert in breast interventions. The techniques utilized were FNA in lesions categorized as BI-RADS 3 as an alternative to short-term follow-up (6 months), CNB in BI-RADS 4a nodules with elastograms with a soft pattern (patterns 2 or 3), and VAB through US in nodules in which the elastogram showed a stiff pattern 4 , which increased the suspicion of malignant nodules.

The samples were sent to Pathological Anatomy Services, where they were analyzed by expert pathologists to determine the etiology of the lesion. The histopathological result was considered as the accurate diagnostic test or gold standard.

\subsection{Statistical Analysis}

The data were analyzed with the statistical program SPSS, version 15.0. The diagnostic sensitivity and specificity of the elastogram was calculated. In addition, the positive predictive value (PPV) and the negative predictive value (NPV) of the elastogram with respect to the histology for each entity were also calculated. For the analytical analysis, $2 \times 2$ contingency tables were utilized, and Pearson's Chi-squared test and Fisher's exact test were applied.

\section{Results}

\subsection{Demographic and Clinical Findings}

A total of 60 patients were recruited, who had nodules $\leq 1 \mathrm{~cm}$ ( $\mathrm{n}=69$ nodules); of these, three patients were excluded, with four nodules categorized as BI-RADS 3 , who opted for the US follow-up after 6 months. Of the 57 patients left, the average age was $45.6 \pm 11.9$ with an age range from 19 to 70 years old; 65 nodules were included, with an average size of $7.5 \pm 1.7 \mathrm{~mm}$ (range: $4-10 \mathrm{~mm}$ ). Of the 65 nodules, 53 were categorized 
as BI-RADS 3, and 12 as BI-RADS 4a through a B-mode ultrasound. Through the use of elastography, the distribution of the patterns were: cyst artifact $(n=13)$, pattern $1(n=4)$, pattern $2(n=31)$, pattern $3(n=13)$, and pattern $4(n=4)$. The BI-RADS 3 lesions $(n=53)$ were subjected to FNA, whereas a CNB was performed with the BI-RADS 4a lesions that showed elastography patterns 1,2 , or $3(n=8)$. The pattern 4 lesions were subjected to $\operatorname{VAB}(\mathrm{n}=4)$. The findings and the management algorithm are shown in Figure 2 . All the patients with a benign diagnosis were subjected to a follow-up US 3 months later, and the stability of the findings was observed and the patients with a malignant diagnosis were subjected to magnetic resonance imaging for cancer staging, after which the appropriate treatment was provided.

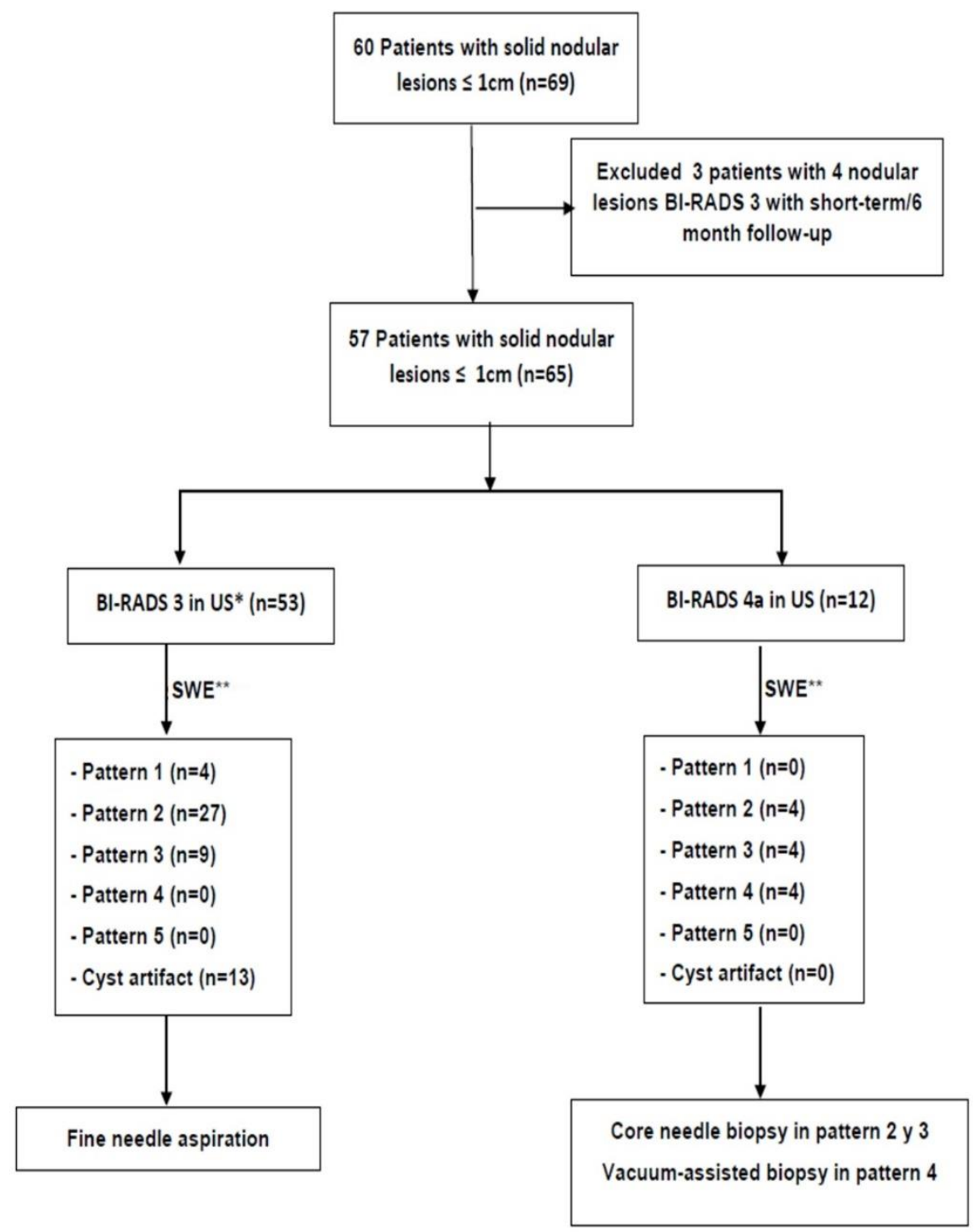

Figure 2. Management algorithm. * Ultrasound. ** Shear wave elastography. BI-RADS—Breast Imaging Reporting and Data System (BI-RADS).

Of the 65 lesions, (Table 1), 15 were cysts, 46 were benign lesions, and three were malignant lesions (two ductal carcinomas in situ, and an infiltrating ductal carcinoma). 
Table 1. Histopathological findings of the 65 lesions.

\begin{tabular}{ccc}
\hline Histopathological Diagnosis & N (65) & $\%$ \\
\hline Cyst & 15 & $23.1 \%$ \\
Fatty lobule & 6 & $9.2 \%$ \\
Epithelial lesions & 41 & $61.5 \%$ \\
Malignant lesions & 3 & $6.2 \%$ \\
\hline
\end{tabular}

3.2. Correlation of the Histopathological Findings with the Ultrasonography and Elastography Findings

Of the 13 nodules which showed a cyst artifact in the elastography, all of them were categorized as BI-RADS 3; the histopathological result was that 12 corresponded to a cyst, and one to benign lesions. Of the four nodules with elastography pattern 1 (BI-RADS 3), all of them obtained benign histopathological results. Of the 31 nodules with elastography pattern 2 (27 BI-RADS 3, and four BI-RADS 4a), five corresponded to cysts, and the rest to benign histopathological lesions, as shown in Table 2. The 13 nodules with elastography pattern 3 (nine BI-RADS 3 and four BI-RADS 4a) obtained benign biopsies. Lastly, of the four nodules with elastography pattern 4 (all BI-RADS 4a), two of them corresponded to a ductal carcinoma in situ, another to an infiltrating ductal carcinoma, and the fourth was a benign lesion (fibroadenoma).

Table 2. Histological correlation with the elastography patterns of the lesions evaluated in the study.

\begin{tabular}{|c|c|c|c|c|}
\hline & & \multicolumn{2}{|c|}{ Histopathological Result } & \multirow[t]{2}{*}{ Total } \\
\hline & & Malignant & Benign & \\
\hline \multirow{2}{*}{ Elastography patterns } & Stiff (pattern 4$)$ & 3 & 1 & 4 \\
\hline & Soft (patterns 1, 2, and 3) and cyst artifact & 1 & 60 & 61 \\
\hline Total & & 4 & 61 & 65 \\
\hline
\end{tabular}

\subsection{Diagnostic Performance of the Elastography}

The predictive values of the elastography were $75 \%$ of sensitivity, $98.36 \%$ of specificity with a correct diagnosis in $96.92 \%$ of the cases $(n=63)$; the PPV was $75 \%$, and the NPV was $98.36 \%$. No significant differences were found between the BI-RADS classification and the elastography patterns $(p=0.85)$. The malignant lesions were associated with stiff elastography patterns (pattern 4), with a $p=0.04$, whereas the benign lesions were associated with soft elastography patterns (patterns 1,2 , and 3, and cyst artifact), with $p=0.03,0.02,0.04$, and 0.02 , respectively. In the diagnosis of the cystic lesions, a correlation was observed between the elastography diagnosis and the histopathological diagnosis, with statistical significance $(p<0.001)$. The sensitivity was $66.7 \%$ and the specificity was 94\%. The PPV was $76.9 \%$ and the NPV was $90.4 \%$.

As for the improvement in the diagnostic precision-which implies adding elastography to the B-mode ultrasound-in three out of four cases categorized as BI-RADS 4a in B-mode, and which showed stiff elastogram results (pattern 4), the result was malignant in the pathological anatomy, whereas for the rest of the softer patterns $(1,2,3$, and cyst artifact), they all showed a benign histopathological result.

\section{Discussion}

This study provides evidence that elastography added to the B-mode in the evaluation of subcentimeter nodules implies an increase in the number of patients who are correctly diagnosed (96.92\%). This is shown by the elastography of patients with a cyst artifact, which obtained a high probability that it would be a cyst and that further follow-up or an invasive diagnostic test would not be needed, whereas on the other hand, lesions with stiff patterns would increase the diagnostic suspicion, which would demand a histopathological diagnosis (75\% of successes). Many authors [13-16] have shown the usefulness of the 
combination of ultrasonography and elastography for the differentiation of benign and malignant lesions, which should increase the diagnostic precision and could contribute to the improvement of the BI-RADS classification. However, there is a scarce number of studies that have analyzed small-sized lesions in an isolated manner, and these tend to be focused on quantitative elastography SWE [13-15,17]. In this sense, it should be pointed out that the literature shows the efficiency of different elastography techniques for the differential diagnosis of solid bodies in breasts by considering their size [10]. However, other authors [9-11] have reported on the contribution of SWE to the diagnosis of breast cancer in lesions $<2 \mathrm{~cm}$. Our study also supports the usefulness of elastography for the early detection of malignant lesions $\leq 1 \mathrm{~cm}$, and up to the present, it is the only study in the literature in which lesions that are so small in size have been analyzed.

In addition to semiquantitative elastography (SWE), the significant association between the diagnosis based on the elastography pattern and the histopathological diagnosis of all the benign solid nodules $\leq 1 \mathrm{~cm}(p<0.05)$ is evident in our results. These findings are in agreement with those reported in the literature, which have shown the contribution of the association between ultrasonography and elastography for the differentiation between benign and malignant lesions $[13,18]$. With respect to the cyst artifact, an association was demonstrated between it and the histopathological diagnosis of the cystic lesions $(p<0.02)$, with a high specificity $(94 \%)$. These findings are in agreement with those obtained by Barr [19]. Keeping in mind that cystic nodules $<1 \mathrm{~cm}$ are benign lesions, it can be assumed that the detection of this artifact would be diagnostic, and therefore unnecessary punctures would not be needed.

Elastography is defined as a decisive test for the diagnosis of benign or malignant subcentimeter nodules, with a correct diagnosis in a high number of cases $(96.92 \%)$. Therefore, when faced with B-mode images categorized as having a low suspicion of malignancy and elastograms with a cyst artifact, we could category them as BI-RADS 2; if the elastograms were soft (patterns 1,2, or 3), we could say that we are dealing with lesions with a high probability of being benign (BI-RADS 3), whereas lesions with more stiff elastograms (patterns 4 or 5) would necessarily have to be biopsied (BI-RADS 4a or b), as already proposed by other authors [20]. The management algorithm proposed is shown in Figure 3.

The present study had some limitations, among which we can find that the study was retrospective in nature, and the sample size was small. In addition, as the sample came from a single center, which is a referral center, a selection bias could be present. On the other hand, we could not evaluate the interobserver or intraobserver variability in the acquisition of data and their interpretation. It was also not possible to independently evaluate the US image of the elastogram pattern, as the entire study was conducted by the same radiologist in a single intervention. Lastly, the results of our study need to be validated with multi-center prospective studies that utilize a larger sample size with a greater diversity of the sample. 


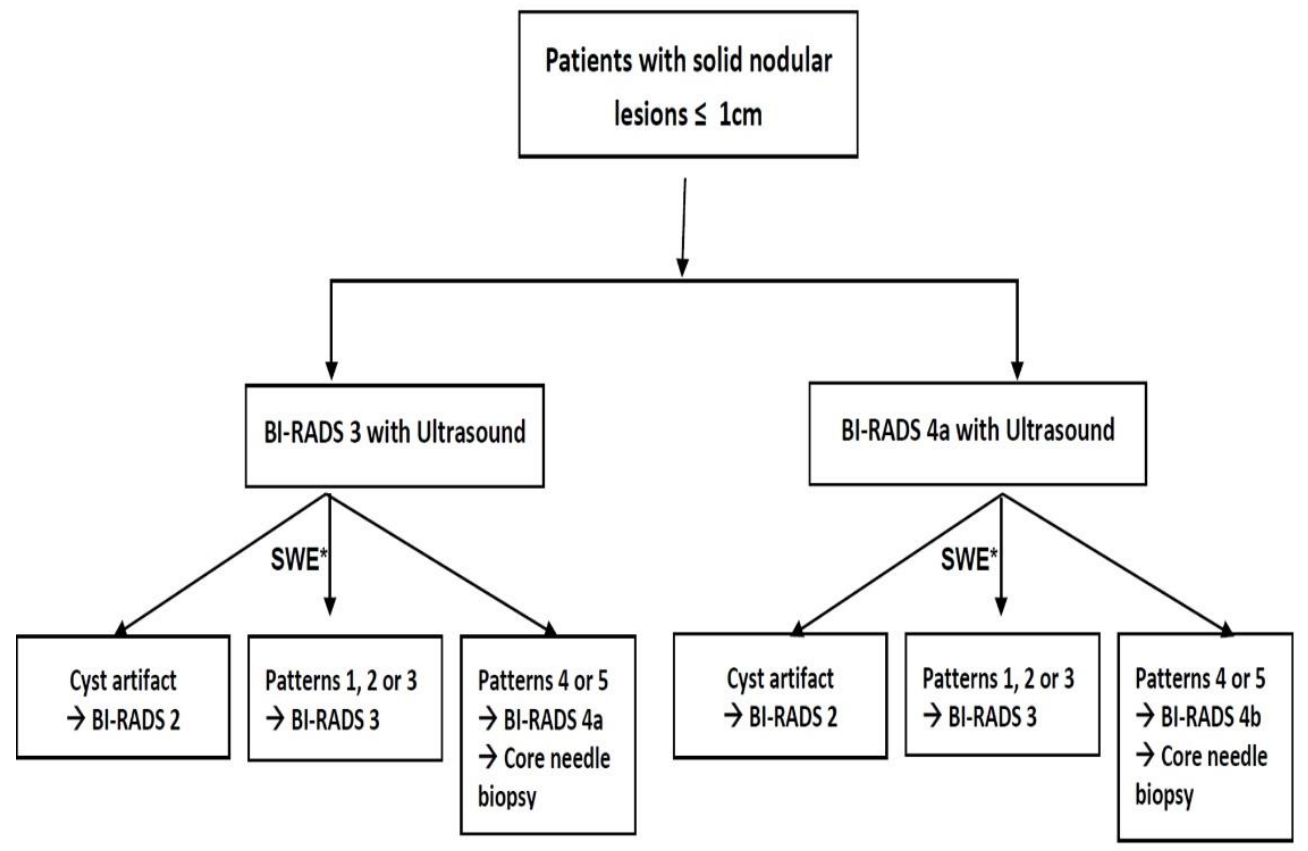

Figure 3. Management algorithm proposed. * Shear wave elastography.

\section{Conclusions}

Elastography added to the B-mode ultrasound of subcentimeter lesions increases the diagnostic precision and could reduce the BI-RADS category of lesions with a cyst artifact, re-categorizing them as BI-RADS 2 (downgrade), or if they obtained soft elastograms to BI-RADS 3. Furthermore, lesions with stiff elastograms (patterns 4 or 5) require a biopsy, even when the B-mode US morphology indicates a benign lesion (upgrade).

Author Contributions: Conceptualization: F.G.-A., Y.M.-P., J.d.D.B.-S., A.A.G.-O., and M.A.; data curation: F.G.-A., Y.M.-P., and J.d.D.B.-S.; methodology: F.G.-A., Y.M.-P., J.d.D.B.-S., and J.d.D.B.-M.; formal analysis: F.G.-A., M.A., Y.M.-P., J.d.D.B.-S., J.d.D.B.-M. and A.A.G.-O.; resources: F.G.-A., M.A., and J.d.D.B.-S.; visualization: F.G.-A. and J.d.D.B.-S.; writing-review and editing, all authors. All authors have read and agreed to the published version of the manuscript.

Funding: This research received no external funding.

Institutional Review Board Statement: The study was conducted according to the guidelines of the Declaration of Helsinki, and approved by the Institutional Review Board of our hospital (Hospital Clínico Universitario "Virgen de la Arrixaca").

Informed Consent Statement: Informed consent was obtained from all subjects involved in the study.

Data Availability Statement: The data used in this study are found in the medical records of the patients examined. They re-quire a specific authorization for their review.

Conflicts of Interest: The authors declare no conflict of interest.

\section{References}

1. Gennisson, J.L.; Deffieux, T.; Fink, M.; Tanter, M. Ultrasound elastography: Principles and techniques. Diagn. Interv. Imaging 2013, 94, 487-495. [CrossRef] [PubMed]

2. Youk, J.H.; Gweon, H.M.; Son, E.J. Shear-wave elastography in breast ultrasonography: The state of the art. Ultrasonography 2017, 36, 300-309. [CrossRef] [PubMed]

3. Barr, R.G. Sonographic breast elastography: A primer. J. Ultrasound Med. 2012, 31, 773-783. [CrossRef] [PubMed]

4. Barr, R.G. Elastography in clinical practice. Radiol. Clin. N. Am. 2014, 52, 1145-1162. [CrossRef]

5. Carlsen, J.; Ewertsen, C.; Sletting, S.; Vejborg, I.; Schäfer, F.K.; Cosgrove, D.; Bachmann Nielsen, M. Ultrasound Elastography in Breast Cancer Diagnosis. Ultraschall Med. 2015, 36, 550-562. [CrossRef] 
6. $\quad$ Lee, S.H.; Cho, N.; Chang, J.M.; Koo, H.R.; Kim, J.Y.; Kim, W.H.; Bae, M.S.; Yi, A.; Moon, W.K. Two-view versus single-view shear-wave elastography: Comparison of observer performance in differentiating benign from malignant breast masses. Radiology 2014, 270, 344-353. [CrossRef] [PubMed]

7. D’Orsi, C.J.; Sickles, E.A.; Mendelson, E.B.; Morris, E.A. ACR BI-RADS®Atlas, Breast Imagin Reporting and Data System. Am. Coll. Radiol. 2013.

8. Barr, R.G.; Destounis, S.V.; Lackey, L.B.; Svensson, W.E.; Balleyguier, C.; Smith, C. Evaluation of breast lesions using sonographic elasticity imaging. J. Ultrasound Med. 2012, 31, 281-287. [CrossRef] [PubMed]

9. Choi, H.Y.; Seo, M.; Sohn, Y.M.; Hwang, J.H.; Song, E.J.; Min, S.Y.; Kang, H.J.; Han, D.Y. Shear wave elastography for the diagnosis of small $(\leq 2 \mathrm{~cm})$ breast lesions: Added value and factors associated with false results. Br. J. Radiol. 2019, 92, 20180341. [CrossRef] [PubMed]

10. Bai, M.; Zhang, H.P.; Xing, J.F.; Shi, Q.S.; Gu, J.Y.; Li, F.; Chen, H.L.; Zhang, X.M.; Fang, Y.; Du, L.F. Acoustic Radiation Force Impulse Technology in the Differential Diagnosis of Solid Breast Masses with Different Sizes: Which Features Are Most Efficient? BioMed Res. Int. 2015, 2015, 410560. [CrossRef] [PubMed]

11. Kim, S.J.; Ko, K.H.; Jung, H.K.; Kim, H. Shear Wave Elastography: Is It a Valuable Additive Method to Conventional Ultrasound for the Diagnosis of Small ( $\leq 2 \mathrm{~cm}$ ) Breast Cancer? Medicine 2015, 94, e1540. [CrossRef] [PubMed]

12. Itoh, A.; Ueno, E.; Tohno, E.; Kamma, H.; Takahashi, H.; Shiina, T.; Yamakawa, M.; Matsumura, T. Breast disease: Clinical application of US elastography for diagnosis. Radiology 2006, 239, 341-350. [CrossRef] [PubMed]

13. Wojcinski, S.; Boehme, E.; Farrokh, A.; Soergel, P.; Degenhardt, F.; Hillemanns, P. Ultrasound real-time elastography can predict malignancy in BI-RADS(R)- US 3 lesions. BMC Cancer 2013, 13, 159. [CrossRef] [PubMed]

14. Thomas, A.; Fischer, T.; Frey, H.; Ohlinger, R.; Grunwald, S.; Blohmer, J.U.; Winzer, K.J.; Weber, S.; Kristiansen, G.; Ebert, B.; et al. Real-time elastography-an advanced method of ultrasound: First results in 108 patients with breast lesions. Ultrasound Obstet. Gynecol. 2006, 28, 335-340. [CrossRef] [PubMed]

15. Li, D.D.; Xu, H.X.; Guo, L.H.; Bo, X.W.; Li, X.L.; Wu, R.; Xu, J.M.; Zhang, Y.F.; Zhang, K. Combination of two-dimensional shear wave elastography with ultrasound breast imaging reporting and data system in the diagnosis of breast lesions: A new method to increase the diagnostic performance. Eur. Radiol. 2016, 26, 3290-3300. [CrossRef] [PubMed]

16. Hao, S.Y.; Jiang, Q.C.; Zhong, W.J.; Zhao, X.B.; Yao, J.Y.; Li, L.J.; Luo, B.M.; Ou, B.; Zhi, H. Ultrasound Elastography Combined With BI-RADS-US Classification System: Is It Helpful for the Diagnostic Performance of Conventional Ultrasonography? Clin. Breast. Cancer 2016, 16, e33-e41. [CrossRef] [PubMed]

17. Ignee, A.; Jenssen, C.; Arcidiacono, P.G.; Hocke, M.; Möller, K.; Saftoiu, A.; Will, U.; Fusaroli, P.; Iglesias-Garcia, J.; Ponnudurai, R.; et al. Endoscopic ultrasound elastography of small solid pancreatic lesions: A multicenter study. Endoscopy 2018, 50, 1071-1079. [CrossRef] [PubMed]

18. Vinnicombe, S.J.; Whelehan, P.; Thomson, K.; McLean, D.; Purdie, C.A.; Jordan, L.B.; Hubbard, S.; Evans, A.J. What are the characteristics of breast cancers misclassified as benign by quantitative ultrasound shear wave elastography? Eur. Radiol. 2014, 24, 921-926. [CrossRef] [PubMed]

19. Barr, R.G.; Lackey, A.E. The utility of the "bull's-eye" artifact on breast elasticity imaging in reducing breast lesion biopsy rate. Ultrasound Q. 2011, 27, 151-155. [CrossRef] [PubMed]

20. Han, J.; Li, F.; Peng, C.; Huang, Y.; Lin, Q.; Liu, Y.; Cao, L.; Zhou, J. Reducing Unnecessary Biopsy of Breast Lesions: Preliminary Results with Combination of Strain and Shear-Wave Elastography. Ultrasound Med. Biol. 2019, 45, 2317-2327. [CrossRef] [PubMed] 